hep-ph/0409273

KEK-TH-981

\title{
Top Spin Correlations in Theories with Large Extra-Dimensions at the Large Hadron Collider
}

\author{
Masato Arai ${ }^{a *}$, Nobuchika Okada ${ }^{b \dagger}$, Karel Smolek ${ }^{c} \ddagger$ \\ and \\ Vladislav Šimák ${ }^{a, d} \S$ \\ ${ }^{a}$ Institute of Physics, AS CR, 182 21, Prague 8, Czech Republic \\ ${ }^{b}$ Theory Division, KEK, Tsukuba, Ibaraki, 305-0801, Japan \\ ${ }^{c}$ Institute of Experimental and Applied Physics, \\ Czech Technical University in Prague, 128 00, Prague 2, Czech Republic \\ ${ }^{d}$ Faculty of Nuclear Sciences and Physical Engineering, \\ Czech Technical University in Prague, 115 19, Prague 1, Czech Republic
}

\begin{abstract}
In theories with large extra dimensions, we study the top spin correlations at the Large Hadron Collider. The $s$-channel process mediated by graviton Kaluza-Klein modes contributes to the top-antitop pair production in addition to the Standard Model processes, and affects the resultant top spin correlations. We calculated the full density matrix for the top-antitop pair production. With the fundamental scale of the extra dimensional theory below $2 \mathrm{TeV}$, we find a sizable deviation of the top spin correlations from the Standard Model one.
\end{abstract}

*arai@fzu.cz

†okadan@post.kek.jp

†karel.smolek@utef.cvut.cz

§simak@fzu.cz 


\section{Introduction}

Recently, the possibility that our space has more than three spatial dimensions has been vigorously studied [1, 2]. Typical setup is that the standard model (SM) fields reside on $3+1$ dimensional manifold called "3-brane" embedded in higher dimensional space-time while only graviton can propagate into the whole space-time dimensions. This setup opens up a new way to solve the gauge hierarchy problem, namely, a huge hierarchy between the electroweak scale and the 4-dimensional Planck scale, as addressed by Arkani-Hamed, Dimopoulos and Dvali (ADD) 1]. In their model, 4-dimensional Planck scale $M_{p l}$ can be expressed by the fundamental scale of $4+n$ dimensions $M_{D}$ and the common radius of the compactified $n$ extra dimensions $R$ such as $M_{p l}=M_{D}\left(M_{D} R\right)^{n / 2}$. If the compactification radius is large enough (for example, $R \sim 0.1 \mathrm{~mm}$ for $n=2), M_{D}$ can be $\mathcal{O}(1 \mathrm{TeV})$ and thus the gauge hierarchy problem can be solved.

Effects of extra dimensions are recast in four dimensional effective theory valid below $M_{D}$ [3. 4]. In the effective theory, the graviton propagating in the whole $4+n$ dimensions can be expressed as an infinite tower of Kaluza-Klein (KK) modes which contain spin-2 (KK-graviton), spin-1 (gravi-vector) and spin-0 (gravi-scalar) excitations. The KK gravitons and the graviscalars couple to the energy-momentum tensor of the SM fields and its trace, respectively, while the gravi-vector has no interaction with them. Since the trace of the energy-momentum tensor is vanishing for massless fields, we can ignore the gravi-scalar interaction at high energy processes. Although each vertex between the SM fields and the KK gravitons are suppressed by $M_{p l}$, the effective coupling is enhanced due to contributions of a large number of KK gravitons so as to be suppressed by $M_{D}=\mathcal{O}(1 \mathrm{TeV})$. Therefore, new phenomena induced by the $\mathrm{KK}$ gravitons, for example, the direct KK graviton emission processes and the virtual KK graviton exchange processes, can be expected at high energy collisions [5]. In particular, the virtual KK graviton exchange process is interesting, since it can give rise to characteristic spin configurations and angular distributions for outgoing particles, which reflect the spin-2 nature of the intermediate KK gravitons.

The top-antitop quark pair is a good candidate to study its spin correlations, since the top quark, with mass in the range of $175 \mathrm{GeV}$ [6], decays electroweakly before hadronizing [7], and thus the possible polarization of the top-antitop quark pair is transferred to its decay products. 
There are thus significant angular correlations among the top quark spin axis and the direction of motion of the decay products.

For the hadronic top-antitop pair production process through the quantum chromodynamics (QCD) interaction, the spin correlations have been extensively studied [8, 9, 10]. It is found that there is a spin asymmetry between the produced top-antitop pairs, namely, the number of produced top-antitop quark pairs with both spin up or spin down is different from the number of pairs with the opposite spin combinations. If the top quark is coupled to a new physics beyond the SM, the top-antitop spin correlations could be altered. Therefore, the top-antitop correlations are useful information to test the SM and also a possible new physics at hadron colliders. Note that the Large Hadron Collider (LHC) has a big advantage to study them, since it will produce almost 10 millions top quarks per year.

In the ADD model, there exists a new top-antitop pair production process through the virtual KK graviton exchange in the $s$-channel. The effect of the virtual KK graviton exchange process has been studied for the total cross section of the top-antitop pair production process at hadron collider [11] and for polarized amplitudes at $e^{+} e^{-}$and $\gamma \gamma$ colliders [12, 13]. The purpose of this paper is to examine the effect of the virtual KK graviton exchange process on the spin correlation of the top-antitop pairs produced at the LHC. With the fundamental scale of the ADD model below $2 \mathrm{TeV}$, we find a sizable deviation of the spin asymmetry of produced top-antitop quark pairs from the SM one. Furthermore, since, in practice, information of the spins of the produced top-antitop quarks are measured through the angular correlations among the top spin axis and the direction of motion of the decay products of the top quark, we study them also.

This paper is organized as follows. In Sec. 2, we briefly review the top spin correlations. In Sec. 3, we examine the invariant amplitudes for the polarized top-antitop quark pair production processes, $q \bar{q} \rightarrow t \bar{t}$ and $g g \rightarrow t \bar{t}$, including the process mediated by the virtual KK gravitons in the $s$-channel. We perform numerical analysis in Sec. 4. Sec. 5 is devoted to conclusions. In Appendix, full representation of the density matrix (squared invariant amplitude) for the top-antitop pair production is given. 


\section{Spin correlation}

At hadron collider, the top-antitop quark pair is produced through the processes of quarkantiquark pair annihilation and gluon fusion:

$$
i \rightarrow t+\bar{t}, \quad i=q \bar{q}, g g
$$

The former is the dominant process at the Tevatron, while the latter is dominant at the LHC. The produced top-antitop pairs decay before hadronization takes place. The main decay modes in the SM involve leptonic and hadronic modes:

$$
t \rightarrow b W^{+} \rightarrow b l^{+} \nu_{l}, b u \bar{d}, b c \bar{s}
$$

where $l=e, \mu, \tau$. The differential decay rates to a decay product $f=b, l^{+}, \nu_{l}$, etc. in the top quark rest frame can be parameterized as

$$
\frac{1}{\Gamma} \frac{d \Gamma}{d \cos \theta_{f}}=\frac{1}{2}\left(1+\kappa_{f} \cos \theta_{f}\right),
$$

where $\Gamma$ is the partial decay width of the respective decay channel, $\theta_{f}$ is the angle between the chosen top spin axis and the direction of motion of the decay product $f$, and the coefficient $\kappa_{f}$ is the top-spin analyzing power of a particle $f$. The SM values of $\kappa_{f}$ at tree level have been computed [14, for instance, in the semi-leptonic decay, $\kappa_{l^{+}}=+1$ for the charged lepton, $\kappa_{b}=-0.41$ for the $b$-quark and $\kappa_{\nu_{l}}=-0.31$ for the $\nu_{l}$, respectively. In hadronic decay modes, the role of the charged lepton is replaced by the $d$ or $s$ quark.

The total matrix element squared for the top-antitop pair production (2.1) and their decay channels (2.2) is given by

$$
|\mathcal{M}|^{2} \propto \operatorname{Tr}\left[\rho R^{i} \bar{\rho}\right]=\rho_{\alpha^{\prime} \alpha} R_{\alpha \beta, \alpha^{\prime} \beta^{\prime}}^{i} \bar{\rho}_{\beta^{\prime} \beta}
$$

Here the subscripts denote the top and antitop spin indices, and $R^{i}$ denotes the density matrix corresponding to the production of the on-shell top-antitop quark pair through the process $i$ in (2.1):

$$
R_{\alpha \beta, \alpha^{\prime} \beta^{\prime}}^{i}=\sum_{\text {initial spin }} \mathcal{M}\left(i \rightarrow t_{\alpha} \bar{t}_{\beta}\right) \mathcal{M}^{*}\left(i \rightarrow t_{\alpha^{\prime}} \bar{t}_{\beta^{\prime}}\right)
$$


where $\mathcal{M}\left(i \rightarrow t_{\alpha} \bar{t}_{\beta}\right)$ is the amplitude for the top-antitop pair production. The matrices $\rho$ and $\bar{\rho}$ are the density matrices corresponding to the decays of polarized top and antitop quarks into some final states at the top and antitop rest frame, respectively. In the leptonic decay modes, the matrices $\rho$, which leads to (2.3), can be obtained as (see, for instance, [15])

$$
\rho_{\alpha^{\prime} \alpha}=\mathcal{M}\left(t_{\alpha} \rightarrow b l^{+} \nu_{l}\right) \mathcal{M}^{*}\left(t_{\alpha^{\prime}} \rightarrow b l^{+} \nu_{l}\right)=\frac{\Gamma}{2}\left(1+\kappa_{f} \vec{\sigma} \cdot \vec{q}_{f}\right)_{\alpha^{\prime} \alpha}
$$

where $q_{f}$ is the unit vector of the direction of motion of the decay product $f$. The density matrix for the polarized antitop quark is obtained by replacing $\kappa_{f} \rightarrow-\kappa_{f}$ in (2.6) .

It is clear that the best way to analyze the top-antitop spin correlations is to see the angular correlations of two charged leptons $l^{+} l^{-}$produced by the top-antitop quark leptonic decays. In the following, we consider only the leptonic decay channels. Using (2.4)-(2.6) and integrating over the azimuthal angles of the charged leptons, we obtain the following double distribution [8, 9, 10,

$$
\frac{1}{\sigma} \frac{d^{2} \sigma}{d \cos \theta_{l^{+}} d \cos \theta_{l^{-}}}=\frac{1-\mathcal{A} \kappa_{l^{+}} \kappa_{l^{-}} \cos \theta_{l^{+}} \cos \theta_{l^{-}}}{4}
$$

with $\kappa_{l^{+}}=\kappa_{l^{-}}=1$. Here $\sigma$ denotes the cross section for the process of the leptonic decay modes, and $\theta_{l^{+}}\left(\theta_{l^{-}}\right)$denotes the angle between the top (antitop) spin axis and the direction of motion of the antilepton (lepton) in the top (antitop) rest frame. The coefficient $\mathcal{A}$ denotes the spin asymmetry between the produced top-antitop pairs with like and unlike spin pairs defined as

$$
\mathcal{A}=\frac{\sigma\left(t_{\uparrow} \bar{t}_{\uparrow}\right)+\sigma\left(t_{\downarrow} \bar{t}_{\downarrow}\right)-\sigma\left(t_{\uparrow} \bar{t}_{\downarrow}\right)-\sigma\left(t_{\downarrow} \bar{t}_{\uparrow}\right)}{\sigma\left(t_{\uparrow} \bar{t}_{\uparrow}\right)+\sigma\left(t_{\downarrow} \bar{t}_{\downarrow}\right)+\sigma\left(t_{\uparrow} \bar{t}_{\downarrow}\right)+\sigma\left(t_{\downarrow} \bar{t}_{\uparrow}\right)}
$$

where $\sigma\left(t_{\alpha} \bar{t}_{\dot{\alpha}}\right)$ is the cross section of the top-antitop pair production at parton level with denoted spin indices.

In the SM, at the lowest order of $\alpha_{s}$, the spin asymmetry is found to be $\mathcal{A}=+0.302$ for the LHC. ${ }^{1}$ Since in the ADD model there is a new contribution to the top-antitop pair production process (2.1) through the virtual KK graviton exchange in the $s$-channel, the spin asymmetry (2.8) can be altered from the SM one. In the next section, we calculate the squared amplitudes for the top-antitop pair production including the virtual KK graviton mediated process.

\footnotetext{
${ }^{1}$ The parton distribution function set of CTEQ5M1 [16] has been used in our calculations. The resultant spin asymmetry somewhat depends on the parton distribution functions used.
} 


\section{Scattering Amplitude}

In the 4-dimensional effective theory of the ADD model, the interaction Lagrangian between the $n$-th KK mode of graviton $G^{(\vec{n})}$ and the SM fields is given by

$$
\mathcal{L}_{i n t}=-\frac{1}{M_{P}} \sum_{\vec{n}} G_{\mu \nu}^{(\vec{n})} T^{\mu \nu},
$$

where $M_{P}=M_{p l} / \sqrt{8 \pi}$ is the reduced 4-dimensional Planck scale, and $T^{\mu \nu}$ is energy momentum tensor of the SM fields. Thus, there exists the top-antitop pair production process $\lambda\left(k_{1}\right)+\bar{\lambda}\left(k_{2}\right) \rightarrow$ $t\left(k_{3}\right)+\bar{t}\left(k_{4}\right)$ (where $\lambda$ denotes quark or gluon) through the virtual KK graviton exchange in the $s$-channel. In the 4-dimensional effective theory with energies smaller than the $4+\mathrm{n}$ dimensional Planck scale $M_{D}$, the amplitude for the KK graviton exchange process would be described as 3 .

$$
\mathcal{M}_{G}=\frac{4 \pi \lambda}{M_{D}^{4}} T^{\mu \nu}\left(k_{1}, k_{2}\right) T_{\mu \nu}\left(k_{3}, k_{4}\right) .
$$

All the ambiguity such as the number of extra dimensions and the regularization procedure for the contributions from the infinite number of KK gravitons are encoded by an order one parameter $\lambda$. Hereafter we use the effective scattering amplitude (3.2) for the top-antitop pair production process and consider the two cases $\lambda= \pm 1$. Using (3.2), the amplitudes of the top-antitop pair production process through the virtual KK graviton exchanges can be explicitly described as (color and flavor indices are suppressed)

$$
\begin{aligned}
\mathcal{M}_{G}(q \bar{q} \rightarrow t \bar{t})= & f_{G}\left\{\left[\bar{\psi}\left(k_{2}\right) \gamma^{\mu} \psi\left(k_{1}\right)\right]\left[\bar{\psi}\left(k_{3}\right) \gamma_{\mu} \psi\left(k_{4}\right)\right]\left(k_{1}-k_{2}\right)^{\nu}\left(k_{3}-k_{4}\right)_{\nu}\right. \\
& \left.+\left[\bar{\psi}\left(k_{3}\right) \gamma^{\mu}\left(k_{1}-k_{2}\right)_{\mu} \psi\left(k_{4}\right)\right]\left[\bar{\psi}\left(k_{2}\right) \gamma^{\nu}\left(k_{3}-k_{4}\right)_{\nu} \psi\left(k_{1}\right)\right]\right\} \\
\mathcal{M}_{G}(g g \rightarrow t \bar{t})= & 4 f_{G}\left\{-\left[\psi\left(\bar{k}_{3}\right) \gamma^{\mu}\left(k_{3}-k_{4}\right)_{\mu} \psi\left(k_{4}\right)\right]\left(k_{1} \cdot k_{2}\right)\left[\epsilon\left(k_{1}\right) \cdot \epsilon\left(k_{2}\right)\right]\right. \\
& +\left[\bar{\psi}\left(k_{3}\right) \gamma^{\mu} k_{2 \mu} \psi\left(k_{4}\right)\right]\left[k_{1} \cdot\left(k_{3}-k_{4}\right)\right]\left[\epsilon\left(k_{1}\right) \cdot \epsilon\left(k_{2}\right)\right] \\
+ & {\left[\bar{\psi}\left(k_{3}\right) \gamma^{\mu} k_{1 \mu} \psi\left(k_{4}\right)\right]\left[k_{2} \cdot\left(k_{3}-k_{4}\right)\right]\left[\epsilon\left(k_{1}\right) \cdot \epsilon\left(k_{2}\right)\right] } \\
+ & {\left[\bar{\psi}\left(k_{3}\right) \gamma^{\mu} \epsilon_{\mu}\left(k_{2}\right) \psi\left(k_{4}\right)\right]\left(k_{1} \cdot k_{2}\right)\left[\left(k_{3}-k_{4}\right) \cdot \epsilon\left(k_{1}\right)\right] } \\
& \left.+\left[\bar{\psi}\left(k_{3}\right) \gamma^{\mu} \epsilon_{\mu}\left(k_{1}\right) \psi\left(k_{4}\right)\right]\left(k_{1} \cdot k_{2}\right)\left[\left(k_{3}-k_{4}\right) \cdot \epsilon\left(k_{2}\right)\right]\right\}
\end{aligned}
$$

where $f_{G} \equiv \pi \lambda / 2 M_{D}^{4}$, and $\epsilon\left(k_{i}\right)(i=1,2)$ are the polarization vectors of the initial gluons.

In the center of mass frame, one can straightforwardly calculate the density matrix $R_{\alpha \beta, \alpha^{\prime} \beta^{\prime}}^{i}$ including both the SM (QCD) and the virtual KK graviton contributions. In the following, we 
give the diagonal parts of the density matrix which are relevant for the estimation of (2.7) and (2.8). Full representation of the density matrix is given in Appendix. In our calculation, we have chosen the helicity spin basis useful for the energy range at the LHC, while the off-diagonal basis is suitable for Tevatron [17]. The results are in the following. For the process $q \bar{q} \rightarrow t \bar{t}$, we find

$$
\begin{aligned}
\left|\mathcal{M}\left(q \bar{q} \rightarrow t_{\uparrow} \bar{t}_{\uparrow}\right)\right|^{2} & =\left|\mathcal{M}\left(q \bar{q} \rightarrow t_{\downarrow} \bar{t}_{\downarrow}\right)\right|^{2} \\
& =\frac{g_{s}^{4}}{9}\left(1-\beta^{2}\right) \sin ^{2} \theta+\frac{f_{G}^{2} s^{4} \beta^{2}}{2}\left(1-\beta^{2}\right) \sin ^{2} 2 \theta, \\
\left|\mathcal{M}\left(q \bar{q} \rightarrow t_{\uparrow} \bar{t}_{\downarrow}\right)\right|^{2} & =\left|\mathcal{M}\left(q \bar{q} \rightarrow t_{\downarrow} \bar{t}_{\uparrow}\right)\right|^{2} \\
& =\frac{g_{s}^{4}}{9}\left(1+\cos ^{2} \theta\right)+\frac{f_{G}^{2} s^{4} \beta^{2}}{2}\left(\cos ^{2} 2 \theta+\cos ^{2} \theta\right),
\end{aligned}
$$

where $\theta$ is the scattering angle between the incoming $q$ and outgoing $t, g_{s}$ is the QCD coupling constant, $\beta=\sqrt{1-4 m_{t}^{2} / s}$, and $m_{t}$ is top quark mass. For the $g g$ initial state, we obtain

$$
\begin{aligned}
& \left|\mathcal{M}\left(g g \rightarrow t_{\uparrow} \bar{t}_{\uparrow}\right)\right|^{2}=\left|\mathcal{M}\left(g g \rightarrow t_{\downarrow} \bar{t}_{\downarrow}\right)\right|^{2} \\
& \quad=\frac{g_{s}^{4} \beta^{2}}{96} \mathcal{Y}(\beta, \cos \theta)\left(1-\beta^{2}\right)\left(1+\beta^{2}+\beta^{2} \sin ^{4} \theta\right)+\mathcal{Z}(\beta, \theta, s) f_{G} s^{2} \beta^{2}\left(1-\beta^{2}\right) \sin ^{4} \theta \\
& \left|\mathcal{M}\left(g g \rightarrow t_{\uparrow} \bar{t}_{\downarrow}\right)\right|^{2}=\left|\mathcal{M}\left(q \bar{q} \rightarrow t_{\downarrow} \bar{t}_{\uparrow}\right)\right|^{2} \\
& \quad=\frac{g_{s}^{4} \beta^{2}}{96} \mathcal{Y}(\beta, \cos \theta) \sin ^{2} \theta\left(1+\cos ^{2} \theta\right)+\mathcal{Z}(\beta, \theta, s) f_{G} s^{2} \beta^{2} \sin ^{2} \theta\left(1+\cos ^{2} \theta\right)
\end{aligned}
$$

Here $\mathcal{Y}(\beta, \theta, s)$ and $\mathcal{Z}(\beta, \theta, s)$ are defined by

$$
\mathcal{Y}(\beta, \cos \theta)=\frac{7+9 \beta^{2} \cos ^{2} \theta}{\left(1-\beta^{2} \cos ^{2} \theta\right)^{2}}, \quad \mathcal{Z}(\beta, \theta, s)=\frac{g_{s}^{2}}{4\left(1-\beta^{2} \cos ^{2} \theta\right)}+\frac{3}{4} f_{G} s^{2},
$$

respectively. Note that there is no interference between the SM diagram and the virtual KK graviton exchange diagram in the $q \bar{q}$ initiated process, while there is the non-vanishing interference in the $g g$ initiated process.

With the squared amplitudes in (3.5), (3.6), (3.7) and (3.8), the integrated top-antitop quark pair production cross section can be obtained by using the formula

$$
\begin{array}{r}
\sigma_{t o t}\left(p p \rightarrow t_{\alpha} \bar{t}_{\dot{\alpha}}\right)=\sum_{a, b} \int d x_{1} \int d x_{2} \int d \cos \theta f_{a}\left(x_{1}, Q^{2}\right) f_{b}\left(x_{2}, Q^{2}\right) \\
\times \frac{d \sigma\left(a\left(x_{1} E_{C M S}\right) b\left(x_{2} E_{C M S}\right) \rightarrow t_{\alpha} \bar{t}_{\dot{\alpha}}\right)}{d \cos \theta}
\end{array}
$$

where $f_{a}$ is the parton distribution function for a parton $a, E_{C M S}$ is a center-of-mass energy of a proton, and $Q$ is the virtual momentum transfer. 
As can be seen from the formulas of the squared amplitudes, the cross sections through the virtual KK graviton exchange process grow according to a power of the collider center-of-mass energy, and thus the unitarity will be violated at high energies. ${ }^{2}$ This behavior is shown in Fig. 1, where the total cross sections of the top-antitop pair production through $q \bar{q} \rightarrow t \bar{t}$ and $g g \rightarrow t \bar{t}$ at the parton level, respectively, are depicted as a function of parton center-of-mass energy $\sqrt{s}$ (or, equivalently, invariant mass of the produced top-antitop pair $M_{t \bar{t}}$ ). We can see that the cross section of the ADD model grows rapidly with $\sqrt{s}$, while the SM cross section decreases. As mentioned above, we (should) use the formula of (3.2) at energies lower than $M_{D}$. Therefore, in order to make our analysis conservative, we take into account the contributions from the virtual KK graviton exchange processes only for the center-of-mass energy of colliding partons lower than $M_{D}$, namely $\sqrt{s}=M_{t \bar{t}} \leq M_{D}$.

\section{Numerical Results}

Here we present various numerical results and demonstrate interesting properties of measurable quantities in the ADD model. In the following analysis, we use the parton distribution functions of Ref. [16] (CTEQ5M1) and its numerical implementation in PDFLIB [19] from the CERN Program Library with the constant scale $Q=m_{t}=175 \mathrm{GeV}, N_{f}=5$ and $\alpha_{s}(Q)=0.1074$.

Figs. 2 and 3 show the total cross sections of the top-antitop quark pair production as a function of the scale $M_{D}$ at the LHC with the center-of-mass energy $14 \mathrm{TeV}$. In Fig. 2, we present the plots with and without the cut $M_{t \bar{t}} \leq M_{D}$ for the KK graviton mediated process. In both cases, the cross sections in the ADD model trace the SM line for large $M_{D}$. The total cross section without the cut grows rapidly as the scale $M_{D}$ becomes small. On the other hand, the cross section with the cut traces the SM result for small $M_{D}$. It indicates that our analysis is conservative. In Fig. 3, we show the breakdown of the total cross section into the like $\left(t_{\uparrow} \bar{t}_{\uparrow}+t_{\downarrow} \bar{t}_{\downarrow}\right)$ and the unlike top-antitop $\left(t_{\uparrow} \bar{t}_{\downarrow}+t_{\downarrow} \bar{t}_{\uparrow}\right)$ spin pairs in the ADD model besides the total cross section of the SM and the ADD model results. For the scale $M_{D}$ below $2 \mathrm{TeV}$, we can see sizable deviations from the SM one.

\footnotetext{
${ }^{2}$ See, for example, [18 for discussions on the unitarity in theories with large extra dimensions.
} 
Differential cross section for the top-antitop pair production given by

$$
\frac{d \sigma_{t o t}(p p \rightarrow t \bar{t})}{d \cos \theta}=\sum_{a, b} \int d x_{1} \int d x_{2} f_{a}\left(x_{1}, Q^{2}\right) f_{b}\left(x_{2}, Q^{2}\right) \frac{d \sigma(t \bar{t})}{d \cos \theta}
$$

is shown in Fig. 4 with the scale $M_{D}=1 \mathrm{TeV}$ and $E_{C M S}=14 \mathrm{TeV}$. In $\lambda=1$ case, we can see the large deviation from the SM cross section at large scattering angles. On the other hand, the deviation for the SM cross section is small in $\lambda=-1$ case. This is because a cancellation occurs between the interference term among the SM and the KK graviton mediated processes and the purely KK graviton mediated process.

We are also interested in the dependence of the cross section on the center-of-mass energy of parton system $\sqrt{s}=M_{t \bar{t}}$, which is given by

$$
\frac{d \sigma_{t o t}(p p \rightarrow t \bar{t})}{d \sqrt{s}}=\sum_{a, b} \int_{-1}^{1} d \cos \theta \int_{\frac{s}{E_{C M S}^{2}}}^{1} d x_{1} \frac{2 \sqrt{s}}{x_{1} E_{C M S}^{2}} f_{a}\left(x_{1}, Q^{2}\right) f_{b}\left(\frac{s}{x_{1} E_{C M S}^{2}}, Q^{2}\right) \frac{d \sigma(t \bar{t})}{d \cos \theta}
$$

The results with $M_{D}=1 \mathrm{TeV}$ are shown in Fig. 5. The deviation of the cross section in the ADD model from the one in the SM grows as $s\left(\leq M_{D}\right)$ becomes large, since the interference term between the SM and the KK graviton mediated processes and the squared amplitude of the purely KK graviton mediated process are proportional to $s^{2}$ and $s^{4}$, respectively.

Let us show the results for the spin asymmetry $\mathcal{A}$. In Fig. 6] the spin asymmetry as a function of the scale $M_{D}$ is depicted. We can see sizable deviations from the SM one at the scale below $\sim 2 \mathrm{TeV}$. For the $q \bar{q} \rightarrow t \bar{t}$ and the $g g \rightarrow t \bar{t}$ channels, the spin asymmetries as a function of the scale $M_{D}$ are depicted in Fig. [7. Note that for the $q \bar{q} \rightarrow t \bar{t}$ channel the cross section is independent of the sign of $\lambda$ (see (3.5) and (3.6) $)$. As can be seen in Fig. 6 the total spin asymmetries is dominated by the gluon fusion since the gluon fusion is the dominant process for the top-antitop quark pair production at the LHC.

As an analogy to Fig. 4 and Fig. 5, the spin asymmetries as functions of the scattering angle (Fig. 8) and $\sqrt{s}$ (Fig. 9) are also shown for $M_{D}=1 \mathrm{TeV}$. We can see the similar behaviors of the deviations to that in Fig. 4 and Fig. 5.

Finally, in Fig. 10, we show lego plots of $\cos \theta_{l^{+}}$vs. $\cos \theta_{l^{-}}$for the top-antitop events with spin correlations $\mathcal{A}=0.000$ (a), $\mathcal{A}=0.302$ (b) corresponding to the SM prediction, and $\mathcal{A}=0.147$ 
(c) corresponding to the ADD model prediction with $\lambda=1$ and $M_{D}=1 \mathrm{TeV}$. These three plots would be distinguishable.

\section{Conclusion}

In theories with large extra dimensions, we have studied the production of top-antitop pairs and the top spin correlations at the LHC. There is the new contribution to the top-antitop pair production process mediated by the virtual KK gravitons in the $s$-channel. We have computed the corresponding density matrices and presented the various numerical results based on the resultant density matrices. For the fundamental scale $M_{D}$ lower than around $2 \mathrm{TeV}$, we have found the sizeable deviations of the top-antitop production rate, the spin asymmetry etc. from the ones in the SM.

\section{Acknowledgements}

Numerical results published in this work were computed with the Supercluster of the Computing and Information Centre of the Czech Technical University in Prague. N.O. would like to thank the Abdus Salam International Centre for Theoretical Physics, Trieste, during the completion of this work. The work of N.O. is supported in part by the Grant-in-Aid for Scientific Research (\#15740164) from the Ministry of Education, Culture, Sports, Science and Technology of Japan.

\section{Appendix : Density matrix $R_{\alpha \beta, \alpha^{\prime} \beta^{\prime}}^{i}$}

In this appendix, we give the full representation of the density matrix for the top antitop pair production in the ADD model. For the $q \bar{q} \rightarrow t \bar{t}$ process, we find

$$
\begin{aligned}
R_{\uparrow \uparrow, \uparrow \uparrow}^{q} & =R_{\downarrow \downarrow, \downarrow \downarrow}^{q}=-R_{\uparrow \uparrow, \downarrow \downarrow}^{q}=-R_{\downarrow \downarrow, \uparrow \uparrow}^{q} \\
& =\frac{g_{s}^{4}}{9}\left(1-\beta^{2}\right) \sin ^{2} \theta+\frac{f_{G} s^{4} \beta^{2}}{2}\left(1-\beta^{2}\right) \sin ^{2} 2 \theta, \\
R_{\uparrow \uparrow, \uparrow \downarrow}^{q} & =R_{\uparrow \uparrow, \downarrow \uparrow}^{q}=R_{\uparrow \downarrow, \uparrow \uparrow}^{q}=R_{\downarrow \uparrow, \uparrow \uparrow}^{q}=-R_{\uparrow \downarrow, \downarrow \downarrow}^{q}=-R_{\downarrow \uparrow, \downarrow \downarrow}^{q}=-R_{\downarrow \downarrow, \uparrow \downarrow}^{q}=-R_{\downarrow \downarrow, \downarrow \uparrow}^{q}
\end{aligned}
$$




$$
\begin{aligned}
& =\frac{g_{s}^{4}}{9} \sqrt{1-\beta^{2}} \sin \theta \cos \theta+\frac{f_{G} s^{4} \beta^{2}}{4} \sqrt{1-\beta^{2}} \sin 4 \theta, \\
R_{\uparrow \downarrow, \uparrow \downarrow}^{q} & =R_{\downarrow \uparrow, \uparrow \uparrow}^{q}=\frac{g_{s}^{4}}{9}\left(1+\cos ^{2} \theta\right)+\frac{f_{G} s^{4} \beta^{2}}{2}\left(\cos ^{2} 2 \theta+\cos ^{2} \theta\right), \\
R_{\uparrow \downarrow, \downarrow \uparrow}^{q} & =R_{\downarrow \uparrow, \uparrow \downarrow}^{q}=\frac{g_{s}^{4}}{9}\left(-1+\cos ^{2} \theta\right)-\frac{f_{G} s^{4} \beta^{2}}{2}(1+2 \cos 2 \theta) \sin ^{2} \theta,
\end{aligned}
$$

and, for the $g g \rightarrow t \bar{t}$ process,

$$
\begin{aligned}
R_{\uparrow \uparrow, \uparrow \uparrow}^{g} & =R_{\downarrow \downarrow, \downarrow \downarrow}^{g} \\
& =\left(\frac{g_{s}^{4}}{96} \mathcal{Y}(\beta, \cos \theta)\left(1+\beta^{2}+\beta^{2} \sin ^{4} \theta\right)+\mathcal{Z}(\beta, \theta, s) f_{G} s^{2} \sin ^{4} \theta\right) \beta^{2}\left(1-\beta^{2}\right), \\
R_{\uparrow \uparrow, \downarrow \downarrow}^{g} & =R_{\downarrow \downarrow, \uparrow \uparrow}^{g} \\
& =-\left(\frac{g_{s}^{4}}{96} \mathcal{Y}(\beta, \cos \theta)\left(-1+\beta^{2}+\beta^{2} \sin ^{4} \theta\right)+\mathcal{Z}(\beta, \theta, s) f_{G} s^{2} \sin ^{4} \theta\right) \beta^{2}\left(1-\beta^{2}\right), \\
R_{\uparrow \uparrow, \uparrow \downarrow}^{g} & =R_{\uparrow \uparrow, \downarrow \uparrow}^{g}=R_{\uparrow \downarrow, \uparrow \uparrow}^{g}=R_{\downarrow \uparrow, \uparrow \uparrow}^{g}=-R_{\uparrow \downarrow, \downarrow \downarrow}^{g}=-R_{\downarrow \uparrow, \downarrow \downarrow}^{g}=-R_{\downarrow \downarrow, \uparrow \downarrow}^{g}=-R_{\downarrow \downarrow, \downarrow \uparrow}^{g} \\
& =\left(\frac{g_{s}^{4}}{96} \mathcal{Y}(\beta, \cos \theta) \cos \theta+\mathcal{Z}(\beta, \theta, s) f_{G} s^{2}\right) \beta^{2} \sqrt{1-\beta^{2}} \cos \theta \sin ^{3} \theta, \\
R_{\uparrow \downarrow, \uparrow \downarrow}^{g} & =R_{\downarrow \uparrow, \downarrow \uparrow}^{g}=\left(\frac{g_{s}^{4}}{96} \mathcal{Y}(\beta, \cos \theta)+\mathcal{Z}(\beta, \theta, s) f_{G} s^{2}\right) \beta^{2}\left(1+\cos ^{2} \theta\right) \sin ^{2} \theta, \\
R_{\uparrow \downarrow, \downarrow \uparrow}^{g} & =R_{\downarrow \uparrow, \uparrow \downarrow}^{g}=-\left(\frac{g_{s}^{4}}{96} \mathcal{Y}(\beta, \cos \theta)+\mathcal{Z}(\beta, \theta, s) f_{G} s^{2}\right) \beta^{2} \sin ^{4} \theta,
\end{aligned}
$$

where the functions $\mathcal{Y}(\beta, \cos \theta)$ and $\mathcal{Z}(\beta, \theta, s)$ are defined in (3.9).

\section{References}

[1] N. Arkani-Hamed, S. Dimopoulos and G. Dvali, Phys. Lett. 429B (1998) 263, hep-ph/9803315; I. Antoniadis, N. Arkani-Hamed, S. Dimopoulos and G. Dvali, Phys. Lett. 436B (1998) 257, hep-ph/9804398.

[2] L. Randall and R. Sundrum, Phys. Rev. Lett. 83 (1999) 3370, hep-ph/9905221; Phys. Rev. Lett. 83 (1999) 4690, hep-th/9906064.

[3] G.F. Giudice, R. Rattazzi and J.D. Wells, Nucl. Phys. B544 (1999) 3, hep-ph/9811291.

[4] T. Han, J.D. Lykken and R-J. Zhang, Phys. Rev. D59 (1999) 105006, hep-ph/9811350. 
[5] For a review, see, for example, K. Cheung, hep-ph/0305003 and references therein.

[6] F. Abe, et al., Phys. Lett. 74 (1995) 2626, hep-ex/9503002.

[7] I. Bigi, Y. Dokshitzer, V. Khoze, J. Kühn and P. Zerwas, Phys. Lett. B181 (1986) 157.

[8] T. Stelzer and S. Willenbrock, Phys. Lett. B374 (1996) 169, hep-ph/9512292 A. Brandenburg, Phys. Lett. B388 (1996) 626, hep-ph/9603333 D. Chang, S.C. Lee and A. Soumarokov, Phys. Rev. Lett. 77 (1996) 1218, hep-ph/9512417.

[9] G. Mahlon and S. Parke, Phys. Rev. D53 (1996) 4886, hep-ph/9512264 G. Mahlon and S. Parke, Phys. Lett. B411 (1997) 173, hep-ph/9706304.

[10] A. Brandenburg, Phys. Lett. B388 (1996) 626, hep-ph/9603333 W. Bernreuther, A. Brandenburg, Z.G. Si and P. Uwer, Phys. Rev. Lett. 87 (2001) 242002, hep-ph/0107086, Nucl.Phys. B690 (2004) 81, hep-ph/0403035.

[11] P. Mathews, S. Raychaudhuri and K. Sridhar, Phys. Lett. B450 (1999) 343, hep-ph/9811501; S. Lola, P. Mathews, S. Raychaudhuri and K. Sridhar, "Extra Dimensions : A View From the Top", hep-ph/0010010.

[12] K.Y. Lee, H.S. Song, J. Song and C. Yu, Phys. Rev. D60 (1999) 093002, hep-ph/9905227.

[13] K.Y. Lee, S.C. Park, H.S. Song, J. Song and C. Yu, Phys. Rev. D61 (2000) 074005, hep-ph/9910466.

[14] A. Czarnecki, M. Jezabek and J.H. Kühn, Nucl. Phys. B351 (1991) 507.

[15] W. Bernreuther, O. Nachtmann, P. Overmann and T. Schröder, Nucl. Phys. B388 (1992) 53; B406 (1993) 516; A. Brandenburg and J.P. Ma, Phys. Lett. B298 (1993) 318.

[16] H.L. Lai, et al., Eur. Phys. J. C12 (2000) 375, hep-ph/9903282.

[17] G. Mahlon and Y. Shadmi, Phys. Lett. B387 (1996) 199, hep-ph/9606419.

[18] O.J.P. Éboli, T. Han, M.B. Magro and P.G. Mercadante, Phys. Rev. D61 (2000) 094007, hep-ph/9908358. 
[19] Plothow-Besch, Hartmute, Comput. Phys. Commun. 75 (1993) 396-416, CERN-PPE-92123. 


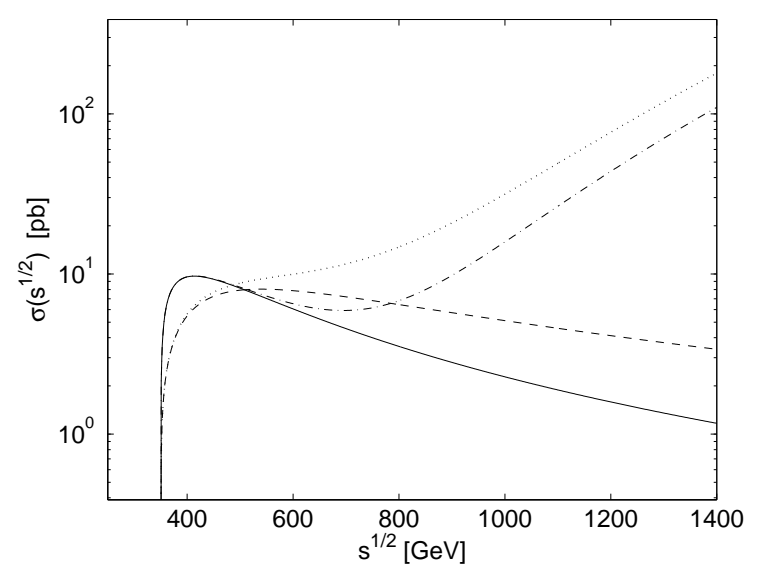

Figure 1: The dependence of the total cross section of the top-antitop quark pair production on the center-of-mass energy of colliding partons. The solid line and the dashed line correspond to the SM prediction for the $q \bar{q} \rightarrow t \bar{t}$ and the $g g \rightarrow t \bar{t}$ channels, respectively. The dash-dotted line and the dotted line correspond to the predictions of the ADD model for the same channels with $\lambda=1$ and $M_{D}=1 \mathrm{TeV}$.

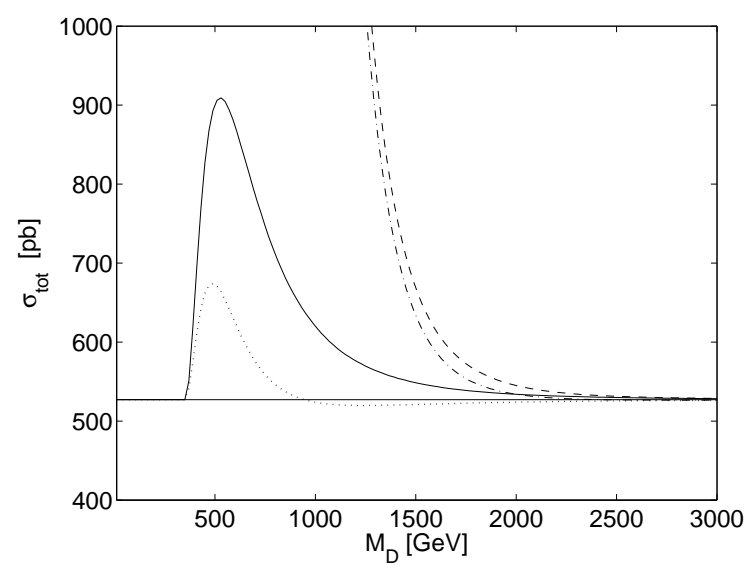

Figure 2: Total cross section for the top pair production as a function of the scale $M_{D}$ for the LHC with center-of-mass energy $14 \mathrm{TeV}$. The horizontal line corresponds to the prediction of the SM, and the solid (dotted) line to the ADD model in $\lambda=1(\lambda=-1)$ case with the cut. The dashed (dash-dotted) line depicts the plot in $\lambda=1(\lambda=-1)$ case without the cut. 


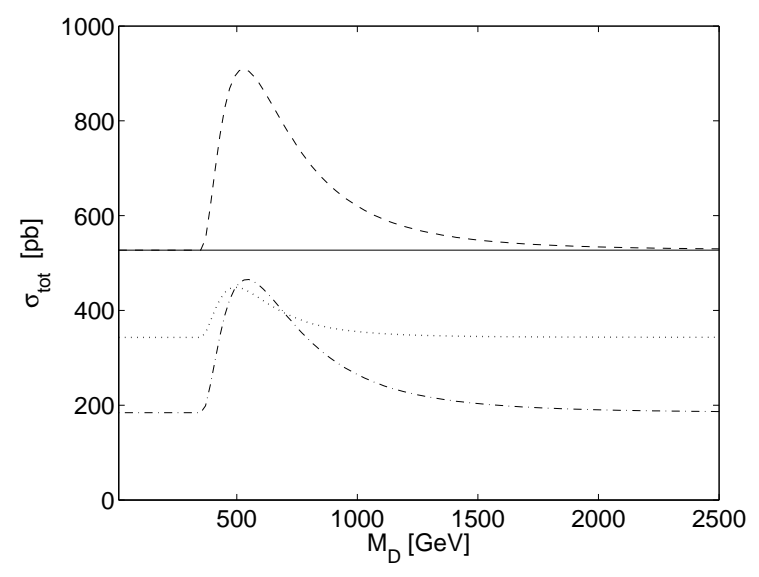

(a) $\lambda=1$

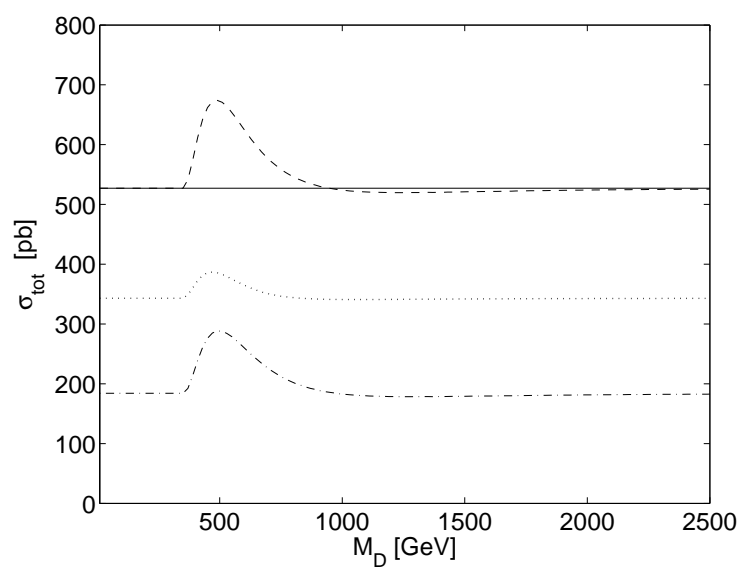

(b) $\lambda=-1$

Figure 3: The breakdown of the total cross section into the like (dotted) and the unlike (dashdotted) top-antitop spin pairs in the ADD model besides the total cross section of the SM (horizontal lines) and the ADD model (dashed lines) results with $M_{D}=1 \mathrm{TeV}$ and $E_{C M S}=14$ $\mathrm{TeV}$.

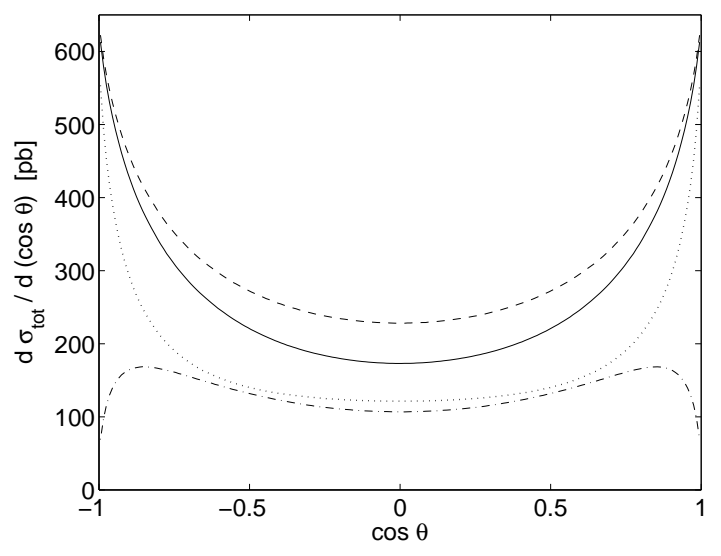

(a) $\lambda=1$

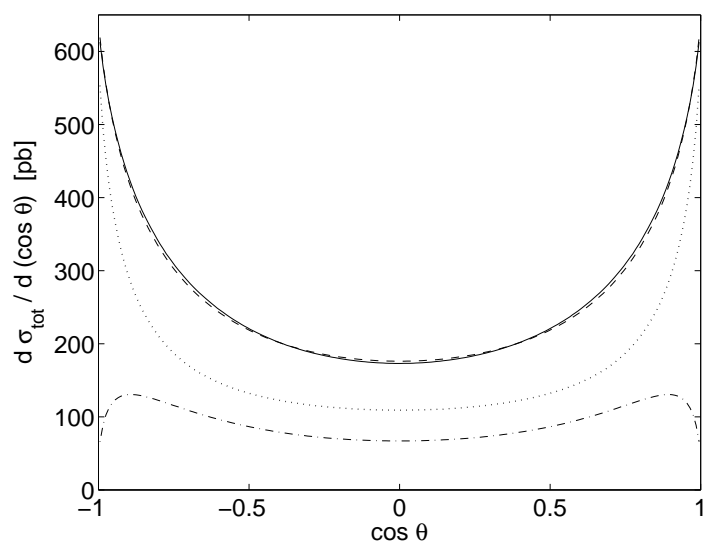

(b) $\lambda=-1$

Figure 4: Differential cross section (4.1) as a function of $\cos \theta$ with $M_{D}=1 \mathrm{TeV}$ and $E_{C M S}=14$ $\mathrm{TeV}$. The solid lines and dashed lines correspond to the results of the SM and the ADD model, respectively. The differential cross sections for the like (dotted) and the unlike (dash-dotted) top-antitop spin pair productions in the ADD model are also depicted. 


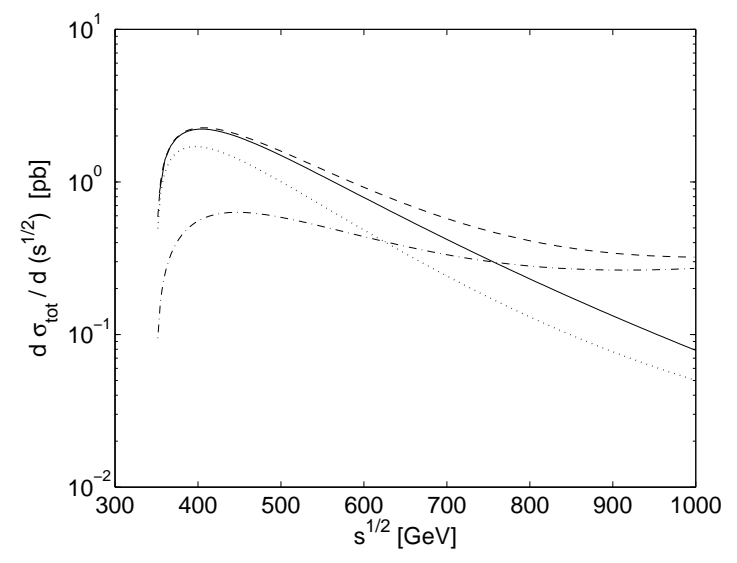

(a) $\lambda=1$

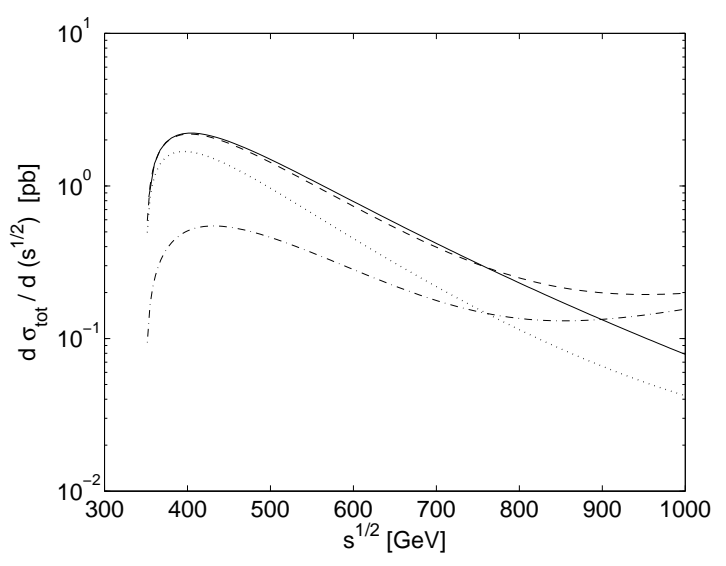

(b) $\lambda=-1$

Figure 5: Differential cross sections (4.2) as a function of the center-of-mass energy of colliding partons $\sqrt{s} \leq M_{D}$. The solid and dashed lines correspond to the results of the SM and the ADD model, respectively. The differential cross sections for the like (dotted) and the unlike (dash-dotted) top-antitop spin pair productions in the ADD model are also depicted.

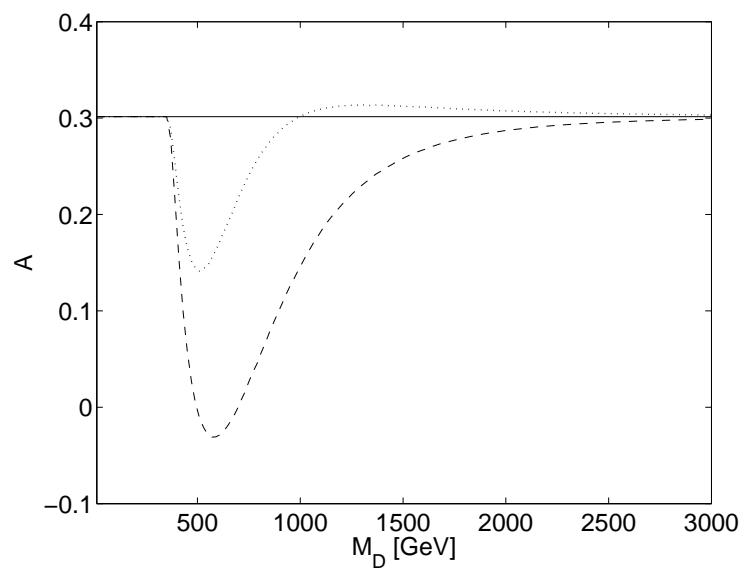

Figure 6: Spin asymmetry $\mathcal{A}$ as a function of the scale $M_{D}$ for the LHC with $E_{C M S}=14 \mathrm{TeV}$. The horizontal line corresponds to the SM. The dashed and the dotted lines correspond to $\lambda=1$ and $\lambda=-1$ cases in the ADD model, respectively. 


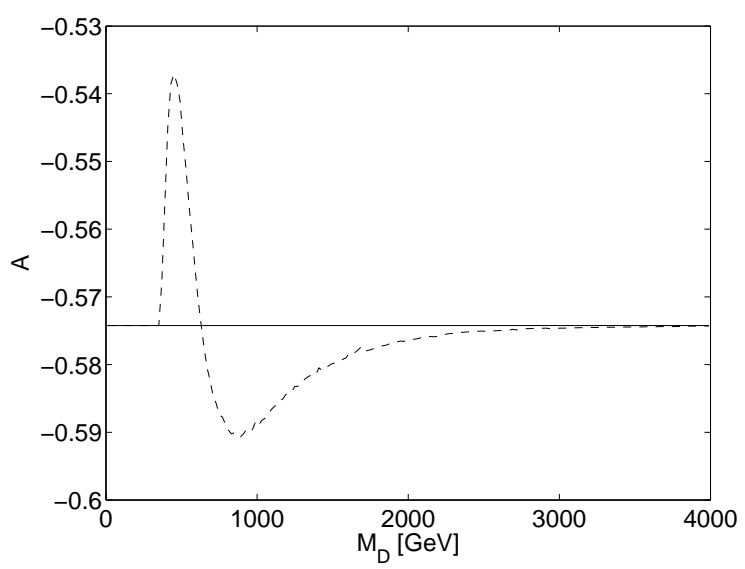

(a)

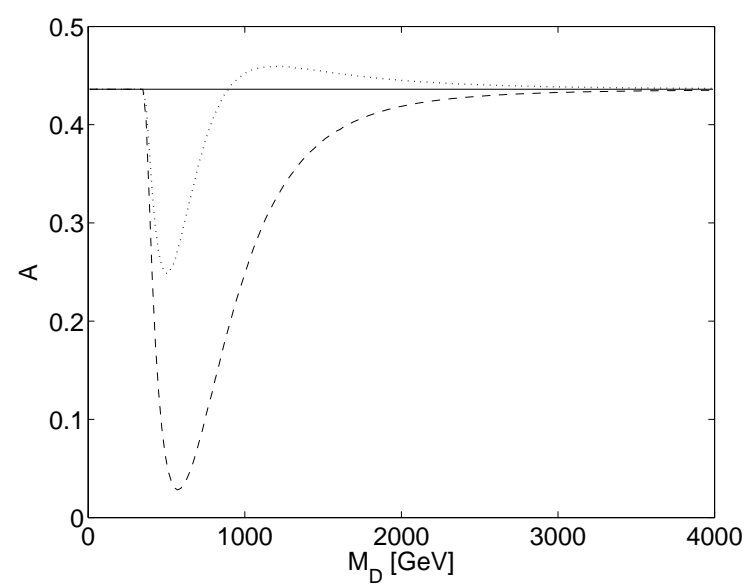

(b)

Figure 7: Spin asymmetry $\mathcal{A}$ for the $q \bar{q} \rightarrow t \bar{t}$ channel (a) and the $g g \rightarrow t \bar{t}$ channel (b) as a function of the scale $M_{D}$ at the LHC with $E_{C M S}=14 \mathrm{TeV}$. The horizontal lines correspond to the SM prediction, while the dashed $(\lambda=1)$ lines and dotted $(\lambda=-1)$ lines correspond to the predictions of the ADD model.

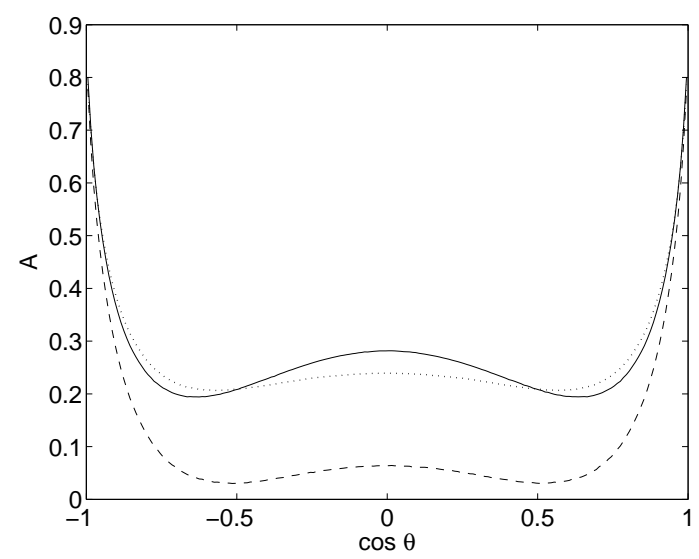

Figure 8: Spin asymmetry $\mathcal{A}$ as a function of the scattering angle $\cos \theta$ with $M_{D}=1 \mathrm{TeV}$ and $E_{C M S}=14 \mathrm{TeV}$. The solid line corresponds to the SM, while the dashed $(\lambda=1)$ line and the dotted $(\lambda=-1)$ line correspond to the results of the ADD model. 


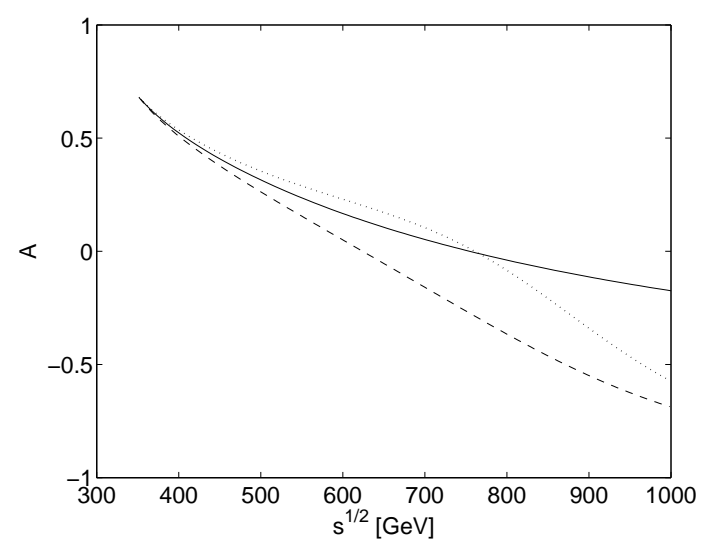

Figure 9: Spin asymmetry $\mathcal{A}$ as a function of the center-of-mass energy of colliding partons $\sqrt{s} \leq M_{D}$ with the scale $M_{D}=1 \mathrm{TeV}$. The solid line corresponds to the SM, while the dashed $(\lambda=1)$ line and the dotted $(\lambda=-1)$ line correspond to the results of the ADD model.

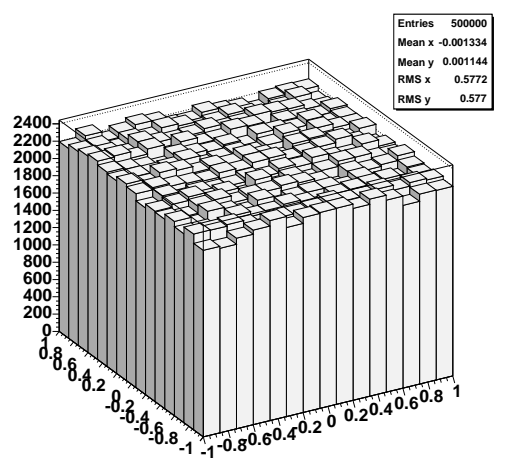

(a)

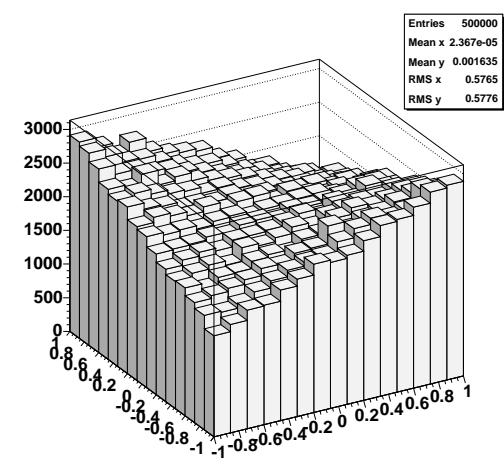

(b)

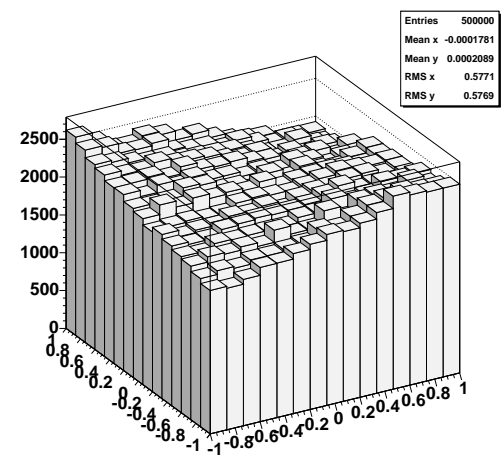

(c)

Figure 10: Lego plots of $\cos \theta_{l^{+}}$vs. $\cos \theta_{l^{-}}$for the top-antitop events with spin correlations $\mathcal{A}=$ 0.000 (a), $\mathcal{A}=0.302$ (b) corresponding to the SM prediction, and $\mathcal{A}=0.147$ (c) corresponding to the ADD model prediction with $\lambda=1, M_{D}=1 \mathrm{TeV}$ and $E_{C M S}=14 \mathrm{TeV}$. 